\title{
Frequency of Medication Non-compliance in Hypertensive Patients Presenting with Stroke: A Case-control Study
}

Vikash Kumar $^{1}$, Barkha Kumari $^{1}$, Eiman Rahat ${ }^{2}$, Sundus Fareed ${ }^{3}$

1. Internal Medicine, Jinnah Sindh Medical University, Karachi, PAK 2. Internal Medicine, Ziauddin University, Karachi, PAK 3. Internal Medicine, Civil Hospital, Karachi, PAK

$\square$ Corresponding author: Sundus Fareed, sundus_fareed@yahoo.com

Disclosures can be found in Additional Information at the end of the article

\section{Abstract \\ Introduction}

Medication compliance (MC) is essential for optimum control and delaying disease progression and complications in chronic illnesses. Patients with hypertension have been repeatedly studied for their pattern of MC in the literature. However, whether or not lack of MC is an issue grave enough to cause medical complications of hypertension is still not clear. The aim of this study was to evaluate if the lack of MC is related to hypertension-related stroke.

\section{Methods}

In this case-control, observational study, 100 hypertensive patients admitted with hypertensive stroke were included. These cases were compared with 200 hypertensive patients without any major hypertensive complication recruited from outpatient clinics. Medication compliance was calculated using the Morisky Medication Adherence Scale (MMAS). Data was entered and analysed using SPSS v. 22.0.

\section{Results}

High compliance patients were more in the control group than the cases (34.5\% vs. $27 \%$ ), similar was with medium ( $41.5 \%$ vs. $30 \%)$. and low compliance patients ( $43 \%$ vs. $24 \%$; $p=0.003)$. In both high compliant and moderate-to-low compliant group, mean systolic and diastolic blood pressure was higher among the cases $(p$ $<0.05)$. Among high compliant patients, cases were taking more pills per day than the controls $(p=0.032)$. Among moderate-to-low compliant patients, $80 \%$ perceived themselves to be highly compliant and only $20 \%$ perceived to be low complaint in the cases, as compared to $60 \%$ controls perceiving themselves compliant and $40 \%$ as low complaint $(p=0.001)$.

\section{Conclusion}

Received 04/26/2019 Review began 04/29/2019 Review ended 05/04/2019 Published 05/07/2019

\section{() Copyright 2019}

Kumar et al. This is an open access article distributed under the terms of the Creative Commons Attribution License CC-BY 3.0., which permits unrestricted use, distribution, and reproduction in any medium, provided the original author and source are credited.
The incidence of low medication adherence is significantly higher in patients with major hypertensive complications such as stroke as compared to hypertensive patients without any major complication.

Categories: Family/General Practice, Internal Medicine, Preventive Medicine

Keywords: morisky medication adherence scale, hypertension, medication compliance, drug adherence, hypertension related stroke, complications of hypertension

\section{Introduction}

Chronic non-communicable diseases are alone responsible for $71 \%$ of global mortality, according to the World Health Organization (WHO). The most common metabolic change predisposing to non-communicable diseases is elevated blood pressure which alone is attributed to $19 \%$ of global deaths related to noncommunicable diseases [1]. "Elevated blood pressure" was defined as systolic pressure 120-129 mm Hg even when diastolic pressure less than $80 \mathrm{~mm} \mathrm{Hg}$ by the American College of Cardiology/American Heart Association (ACC/AHA) High Blood Pressure Guidelines, in 2017. For Stage I and II hypertension, systolic pressure is taken as $130-139 \mathrm{~mm} \mathrm{Hg}$ and $\geqslant 140 \mathrm{~mm} \mathrm{Hg}$ respectively, and diastolic is taken as $80-89 \mathrm{~mm} \mathrm{Hg}$ and $\geqslant 90 \mathrm{~mm} \mathrm{Hg}$, respectively [2]. According to WHO, one in every four adults in Pakistan is hypertensive [3]. Although no nationwide study has been conducted to evaluate the prevalence of hypertension (HTN) in Pakistan, various studies show that it ranges from $26 \%$ to $39 \%$ in adults [4-6].

Since essential hypertension is multi-factorial, its treatment approach is also multi-faceted. Management of HTN involves, adopting a healthy and physically active lifestyle along with add-on drug therapy [7]. As with all other chronic progressive diseases, compliance to a healthy lifestyle and medication regimen are

\section{How to cite this article}

Kumar V, Kumari B, Rahat E, et al. (May 07, 2019) Frequency of Medication Non-compliance in Hypertensive Patients Presenting with Stroke: A Case-control Study. Cureus 11(5): e4605. DOI 10.7759 /cureus.4605 
essential to slow down disease progression and delay the onset of complications [8]. Adherence is not about timely consumption of pills but it reflects an overall healthy lifestyle. In an Ethiopian study, only $23 \%$ of the hypertensive patients were compliant to the lifestyle manifestations advised by their physicians [9]. In a large-scale, longitudinal cohort of hypertensive patients without any cardiac risk factor, there was a 37\% reduced risk of cardiovascular outcome in patients who were compliant to their anti-hypertensive therapy [10].

In another retrospective cohort, as the medication possession ratio reduced in hypertensive patients, their risk of cerebrovascular accidents increased from 1.36-2 times [11]. Compliance to therapy is critical for the prevention of complications in patients with chronic progressive illnesses. Medication non-compliance is a manageable issue. If medication non-compliance actually has a relationship with emergency events in chronic illnesses, addressing non-compliance can achieve greater disease control and reduce the incidence of acute events. We aimed to evaluate if there is any relationship between medication compliance (MC) and cerebrovascular complications among hypertensive patients by comparing MC in patients who have developed a cerebrovascular complication with those who have not.

\section{Materials And Methods \\ Study design, settings, and sample}

It was an observational case-control study conducted in a tertiary care centre in Karachi. Institutional review board approval was attained and written informed consent was attained from all participants. The sample size was calculated using OpenEpi for control to case ratio 2:1 and odds ratio 2 [12]. The sample size for cases was calculated to be 100 and for controls 199.

"Controls" were defined as patients with known hypertension as defined in ACC/AHA guidelines [3]. They were recruited from the outpatient department (OPD) of the hospital. Patients who have had a one or more previous episodes of hypertensive strokes were excluded.

"Cases" were defined as known hypertension patients admitted in the internal medicine department with the clinical and neuro-radiological diagnosis of stroke by the attending physician. Only patients with ischemic or haemorrhagic as a result of raised blood pressure were included. Stroke patients with causes other than raised blood pressure were excluded.

\section{Study instrument}

A structured performa was created. It included two sections. The first section comprised of patient age, gender, duration of diagnosis of hypertension, blood pressure in mmHg and body mass index (BMI) in $\mathrm{kg} / \mathrm{m}^{2}$ (within the OPD for controls and at the time of admission for cases), total number of daily pills taken, selfrated health status (5: excellent, 4: very good, 3: good, 2: fair, 1: poor), and self-rated MC (3: high, 2: moderate, 1 : low).

The second section of the performa comprised Modified Morisky Adherence Scale (MMAS). It is an eightitem validated instrument used to assess the extent of drug adherence. With a maximum score of 8 , it classifies patients into highly compliant (score: 8), moderately compliant (score: $6-<8$ ), and low compliant (score: <6) [13].

\section{Data analysis}

Data was entered and analysed using Statistical Package for Social Sciences (SPSS) version 22.0 (NY, USA). Categorical data was analysed in terms of frequency and percentages. Continuous data were analysed in terms of mean and standard deviation (SD). For the sake of data interpretation, patients who scored $<8$ on MMAS were combined into one group - moderate to low compliant. Similarly, scores 1 and 2 on self-rated MC were combined into one group - moderate to low adherent (score: 1-2) and score 4 and 3 on self-rated health status were combined into one group - very good to good (score: 3-4). The extent of MC as recorded on MMAS was compared with patient factors. Chi-square was applied to find the statistical correlation between the extent of medication compliance and related factors. $P$-value of $\leqslant 0.05$ was considered significant.

\section{Results}

In the control group, there were 92 (46\%) men and 108 (54\%) women. Their mean age was $54.34 \pm 6.07$ years and their mean duration of hypertension was $4.13 \pm 2.03$ years. The mean BMI was $29.94 \pm 2.44 \mathrm{~kg} / \mathrm{m} 2$. Their mean systolic blood pressure was $129.46 \pm 7.25$ and diastolic was $110.33 \pm 4.56$. In the cases group, there were 77 (77\%) men and 23 (23\%) women. Their mean age was $59.47 \pm 3.55$ years and their mean duration of hypertension was $4.43 \pm 1.85$ years. The mean BMI was $29.08 \pm 3.72 \mathrm{~kg} / \mathrm{m} 2$. Their mean systolic blood pressure was $179.50 \pm 8.79$ and diastolic was $123.53 \pm 6.92$. 


\section{Cureus}

The MMAS scores of cases and controls are shown in Table 1. High MC patients were more in the control group than the cases ( $34.5 \%$ vs. $27 \%$ ), similar was with medium MC patients ( $41.5 \%$ vs. $30 \%)$. Low MC patients were common in the cases group ( $43 \%$ vs. $24 \%$ ). The differences were statistically significant ( $p=$ 0.003; Table 1).

\begin{tabular}{|c|c|c|c|c|}
\hline Medication compliance on MMAS & Control group $(n=200)(\%)$ & Cases group $(n=100)(\%)$ & $P$ value & Total patients $(n=300)$ \\
\hline High & $69(34.5 \%)$ & $27(27.0 \%)$ & \multirow{3}{*}{0.003} & $96(32.0 \%)$ \\
\hline Medium & $83(41.5 \%)$ & $30(30.0 \%)$ & & $113(37.7 \%)$ \\
\hline Low & $48(24.0 \%)$ & $43(43.0 \%)$ & & $91(30.3 \%)$ \\
\hline
\end{tabular}

TABLE 1: Extent of medication compliance of cases and controls as assessed by MMAS

In the control group, there were 70 (35\%) patients consuming $1-3$ pills daily, 41 (20.5\%) patients consuming 4-6 pills daily, and 89 (44.5\%) were consuming $>6$ pills per day. There were $24(12 \%)$ patients who self-rated their health as "excellent," 62 (31\%) rated their health as "very good-good," $55(27.5 \%)$ rated their health as "fair," and 59 (29.5\%) rated their health as "poor." There were 136 (68\%) patients who self-rated their drug adherence as "high" and 64 (32\%) self-reported their drug adherence as "moderate-to-low".

In the cases group, there were 18 (18\%) patients consuming 1-3 pills daily, 33 (33\%) patients consuming 4-6 pills daily, and $49(49 \%)$ were consuming $>6$ pills per day. There were $8(8 \%)$ patients who self-rated their health as "excellent," 27 (27\%) rated their health as "very good-good," 31 (31\%) rated their health as "fair," and 34 (34\%) rated their health as "poor." There were 81 (81\%) patients who self-rated their MC as "high" and 19 (19\%) self-reported their MC as "moderate-to-low".

When the cases and controls were divided according to their extent of MC, it was seen that 27 (27\%) cases were high compliant and 73 (73\%) were moderate-to-low compliant. In the control group, 69 (34.5\%) were high compliant and 131 (65.6\%) were moderate-to-low compliant. The factors that can affect the medication compliance are compared to the actual compliance of cases and controls in Table 2. In the cases group of high compliant participants, there were 21 (77.7\%) men and 6 (22.2\%) women as compared to 33 (47.8\%) men and $36(52.2 \%)$ women in the controls group $(p=0.007)$. There was no statistical significance of age and duration of hypertension in both high compliant and moderate-to-low compliant. The mean of both systolic and diastolic blood pressure was statistically significant for both high compliant and moderate-to-low compliant groups. BMI was only statistically significant for men and women in moderate-to-low compliant group. The total number of daily pills was only statistically significant for moderate-to-low compliant group. Self-rated health status did not show any statistically significant relationship with MC. Self-reported MC was statistically significant for moderate-to-low compliant group (Table 2). 


\section{Cureus}

\begin{tabular}{|c|c|c|c|c|c|c|}
\hline & \multicolumn{3}{|c|}{ High medication compliance ( $\mathrm{=}=96 ; 32 \%)$} & \multicolumn{3}{|c|}{ Moderate to low medication compliance $(n=204 ; 68 \%)$} \\
\hline & Cases $(n=27)$ & Controls $(n=69)$ & $\mathrm{p}$ value & Cases $(n=73)$ & Controls $(n=131)$ & $\mathrm{p}$ value \\
\hline \multicolumn{7}{|l|}{ Gender } \\
\hline Male & $21(77.7 \%)$ & $33(47.8 \%)$ & \multirow{2}{*}{0.007} & $56(76.7 \%)$ & $59(45.0 \%)$ & \multirow{2}{*}{0.000} \\
\hline Female & $6(22.2 \%)$ & $36(52.2 \%)$ & & $17(23.3 \%)$ & $72(55.0 \%)$ & \\
\hline \multicolumn{7}{|l|}{ Age } \\
\hline 40-55 years & $11(40.7 \%)$ & $27(39.1 \%)$ & \multirow{2}{*}{0.88} & $40(54.8 \%)$ & $82(62.5 \%)$ & \multirow{2}{*}{0.275} \\
\hline$>56$ years & $16(59.3 \%)$ & $42(60.8 \%)$ & & $33(45.2 \%)$ & $49(37.4 \%)$ & \\
\hline \multicolumn{7}{|c|}{ Duration of hypertension } \\
\hline $0-3$ years & $10(37.0 \%)$ & $35(50.7 \%)$ & \multirow{3}{*}{0.189} & $5(6.8 \%)$ & $18(13.7 \%)$ & \multirow{3}{*}{0.292} \\
\hline 3-6 years & $10(37.0 \%)$ & $26(37.7 \%)$ & & $33(45.2 \%)$ & $59(45.0 \%)$ & \\
\hline$>6$ years & $7(26.0 \%)$ & $8(11.6 \%)$ & & $35(47.9 \%)$ & $54(41.2 \%)$ & \\
\hline \multicolumn{7}{|c|}{ Mean \pm SD blood pressure at admission / in OPD $(\mathrm{mmHg})$} \\
\hline Systolic & $159.85 \pm 11.79$ & $138.24 \pm 6.07$ & $<0.000$ & $184.33 \pm 9.24$ & $130.77 \pm 9.02$ & $<0.000$ \\
\hline Diastolic & $119.25 \pm 7.58$ & $115.22 \pm 7.53$ & 0.020 & $124.82 \pm 10.44$ & $109.23 \pm 12.83$ & $<0.000$ \\
\hline \multicolumn{7}{|c|}{ Mean \pm SD body mass index at admission / in OPD $\left(\mathrm{kg} / \mathrm{m}^{2}\right)$} \\
\hline Males & $34.58 \pm 2.88$ & $34.20 \pm 3.04$ & 0.38 & $33.52 \pm 2.04$ & $32.15 \pm 2.11$ & 0.004 \\
\hline Female & $34.11 \pm 2.77$ & $33.89 \pm 2.53$ & 0.56 & $34.54 \pm 1.55$ & $32.22 \pm 2.74$ & 0.000 \\
\hline \multicolumn{7}{|c|}{ Number of pills consumed per day } \\
\hline $1-3$ & $3(11.1 \%)$ & $24(34.7 \%)$ & \multirow{3}{*}{0.032} & $15(20.5 \%)$ & $46(35.1 \%)$ & \multirow{3}{*}{0.070} \\
\hline $4-6$ & $9(33.3 \%)$ & $11(15.9 \%)$ & & $24(32.8 \%)$ & $30(22.9 \%)$ & \\
\hline$>6$ & $15(55.5 \%)$ & $34(49.3 \%)$ & & $34(46.5 \%)$ & $55(42.0 \%)$ & \\
\hline \multicolumn{7}{|c|}{ Self rated heath status } \\
\hline Excellent & $3(11.1 \%)$ & $7(10.1 \%)$ & \multirow{4}{*}{0.422} & $5(6.8 \%)$ & $17(12.9 \%)$ & \multirow{4}{*}{0.120} \\
\hline Very good - good & $11(40.7 \%)$ & $19(27.5 \%)$ & & $16(22.0 \%)$ & $43(32.8 \%)$ & \\
\hline Fair & $3(11.1 \%)$ & $17(24.6 \%)$ & & $28(38.4 \%)$ & $38(29.0 \%)$ & \\
\hline Poor & $10(37.0 \%)$ & $26(37.8 \%)$ & & $24(32.8 \%)$ & $33(25.2 \%)$ & \\
\hline \multicolumn{7}{|c|}{ Self-rated drug adherence } \\
\hline High & $22(81.4 \%)$ & $58(84.1 \%)$ & \multirow{2}{*}{0.760} & $59(80.8 \%)$ & $78(59.5 \%)$ & \multirow{2}{*}{0.001} \\
\hline Moderate - low & $5(18.5 \%)$ & $11(15.9 \%)$ & & $14(19.2 \%)$ & $53(40.5 \%)$ & \\
\hline
\end{tabular}

\section{TABLE 2: Factors affecting medication compliance compared to the actual compliance of the}

patients as assessed by Modified Morisky Adherence Scale

$\mathrm{SD}$, standard deviation; OPD, outpatient department

\section{Discussion}

In this comprehensive study, hypertensive cases that developed cerebrovascular incident showed significantly reduced medication compliance as compared to the control group. Even in their high compliance group, the cases had higher mean blood pressure and BMI than the controls. Along with this, the 
cases were consuming a higher number of total daily pills. The self-rated health status was comparable in both cases and controls of the high compliant and moderate-to-low compliant groups. In the moderate-tolow compliant group, more cases self-reported their MC to be high as compared to the controls; the difference was statistically significant.

This study is substantial in adding to the growing literature highlighting a crucial relationship between cerebrovascular complications in hypertensive patients who were not compliant to their medications. However, it was a small-sample, single-center study, which took place at one point in time; hence, any casual relationship cannot be deduced. Patients were included in the study when they already have developed the adverse outcome (stroke), hence, this retrospective method is subjected to recall bias. This study assessed medication compliance through a standard, validated research instrument which is a moderately accurate method. More accurate methods of measuring medication compliance including pill counting, prescription record review, electronic monitoring, and directly observed therapy must be applied for more accurate results [14].

In a Chinese study with HTN patients discharged after stroke, 35\% were found to be compliant to their doctor's prescription. Poor MC in their patients was related to female gender, unemployed participants, participants with stage III HTN, participants with poor HTN knowledge, and participants who did not monitor their blood pressure regularly [15]. Comparatively, in this study, the factors associated with moderate-to-low medication compliance in stroke patients were male gender, uncontrolled blood pressure, high BMI, and self-rated high MC.

Results on medication compliance from the REasons for Geographic And Racial Disparities in Stroke (REGARDS) trial also showed low medication compliance in female patients with limited income resources. Patients with low medication compliance in the REGARDS trial had 1.08 times greater risk of stroke [16]. Another study with a similar case-control sample as ours showed that in HTN patients who developed stroke the highest odds ratio (OR) upon logistic regression was seen with "medication not taking as prescribed" (OR =6.07) [17]. Hypertensive patients non-compliant to treatment showed a higher frequency of cerebrovascular accidents as compared to the ones who were compliant (25\% vs. 13\%; OR: $3.04 ; p<0.001$ ) [18]. In a study with hypertensive stroke patients, $17 \%$ were highly compliant of Morisky scale, $6 \%$ were moderately compliant and $77 \%$ had low medication compliance. Their main reasons were lack of knowledge and high cost. Only $3.6 \%$ of patients reported pill burden as a reason to low MC in their study [19].

This study has shown a crucial relationship with reduced medication compliance and the incidence of cerebrovascular accidents in hypertensive patients. Since this study was conducted at one point in time, casual relationship cannot be established. However, we recommend longitudinal studies with larger sample size to study this predictor in depth. Medication non-compliance can be taken as a reversible risk factor for acute events in chronic illnesses. Physicians should maintain a healthy bond with their patients, press the need of medication compliance to their patients, and most importantly aware them about the progression of their disease and consequences of non-compliance. Awareness lets the patients make an informed choice about their attitude towards the disease.

\section{Conclusions}

Hypertension is a chronic illness that requires a multifactorial approach to management. A combination of healthy lifestyle and adequate pharmacologic interventions are essential to keep blood pressures in optimum range and prevent any major organ complications. Patients with major hypertensive complications including stroke are associated with low medication adherence in this study. These patients reported higher BMI and daily pill count and perceived their medication compliance to be higher which actually was not.

\section{Additional Information}

\section{Disclosures}

Human subjects: Consent was obtained by all participants in this study. Dr Ruth Pfau Hospital issued approval 211/11. Animal subjects: All authors have confirmed that this study did not involve animal subjects or tissue. Conflicts of interest: In compliance with the ICMJE uniform disclosure form, all authors declare the following: Payment/services info: All authors have declared that no financial support was received from any organization for the submitted work. Financial relationships: All authors have declared that they have no financial relationships at present or within the previous three years with any organizations that might have an interest in the submitted work. Other relationships: All authors have declared that there are no other relationships or activities that could appear to have influenced the submitted work.

\section{References}

1. Noncommunicable diseases. (2018). Accessed: April 21, 2019: https://www.who.int/news-room/fact- 


\section{Cureus}

sheets/detail/noncommunicable-diseases.

2. Whelton PK, Carey RM, Aronow WS, et al.: 2017

ACC/AHA/AAPA/ABC/ACPM/AGS/APhA/ASH/ASPC/NMA/PCNA guideline for the prevention, detection, evaluation, and management of high blood pressure in adults: a report of the American College of Cardiology/American Heart Association Task Force on Clinical Practice Guidelines. J Am Coll Cardiol. 2018, 71:127-248. 10.1016/j.jacc.2017.11.006

3. Country cooperation strategy at a glance . (2017). Accessed: April 21, 2019: https://apps.who.int/iris/bitstream/handle/10665/136607/ccsbrief_pak_en.pdf;jsessionid=D4B6035B3A3C996BE70A0B

4. Naseem S, Khattak UK, Ghazanfar H, Irfan A: Prevalence of non-communicable diseases and their risk factors at a semi-urban community, Pakistan. Pan Afr Med J. 2016, 23:151. 10.11604/pamj.2016.23.151.8974

5. Shah N, Shah Q, Shah AJ: The burden and high prevalence of hypertension in Pakistani adolescents: a metaanalysis of the published studies. Arch Public Health. 2018, 76:20. 10.1186/s13690-018-0265-5

6. Shafi ST, Shafi T: A survey of hypertension prevalence, awareness, treatment, and control in health screening camps of rural Central Punjab, Pakistan. J Epidemiol Glob health. 2017, 7:135-40. 10.1016/j.jegh.2017.01.001

7. Gupta R, Guptha S: Strategies for initial management of hypertension. Indian J Med Res. 2010, 132:531-42.

8. Kim HS, Cho JH, Yoon KH: New directions in chronic disease management . Endocrinol Metab (Seoul). 2015, 30:159-66. 10.3803/EnM.2015.30.2.159

9. Tibebu A, Mengistu D, Negesa L: Adherence to recommended lifestyle modifications and factors associated for hypertensive patients attending chronic follow-up units of selected public hospitals in Addis Ababa, Ethiopia. Patient Prefer Adherence. 2017, 11:323-30. 10.2147/PPA.S126382

10. Corrao G, Parodi A, Nicotra F, Zambon A, Merlino L, Cesana G, Mancia G: Better compliance to antihypertensive medications reduces cardiovascular risk. J Hypertens. 2011, 29:610-8. 10.1097/HJH.0b013e328342ca97

11. Kim HJ, Yoon SJ, Oh IH, Lim JH, Kim Y: Medication adherence and the occurrence of complications in patients with newly diagnosed hypertension. Korean Circ J. 2016, 46:384-93. 10.4070/kcj.2016.46.3.384

12. OpenEpi. Accessed: April 21, 2019: https://www.openepi.com/SampleSize/SSCC.htm.

13. Okello S, Nasasira B, Muiru AN, Muyingo A: Validity and reliability of a self-reported measure of antihypertensive medication adherence in Uganda. PloS One. 2016, 11:0158499.

10.1371/journal.pone.0158499

14. Vrijens B, Antoniou S, Burnier M, de la Sierra A, Volpe M: Current situation of medication adherence in hypertension. Front Pharmacol. 2017, 8:100. 10.3389/fphar.2017.00100

15. Pan J, Lei T, Hu B, Li Q: Post-discharge evaluation of medication adherence and knowledge of hypertension among hypertensive stroke patients in northwestern china. Patient Prefer Adherence. 2017, 11:1915-22. 10.2147/PPA.S147605

16. Cummings DM, Letter AJ, Howard G, et al.: Medication adherence and stroke/TIA risk in treated hypertensives: results from the REGARDS study. J Am Soc Hypertens. 2013, 7:363-9.

10.1016/j.jash.2013.05.002

17. Baune BT, Aljeesh YI, Bender R: The impact of non-compliance with the therapeutic regimen on the development of stroke among hypertensive men and women in Gaza, Palestine. Saudi Med J. 2004, 25 :

18. Braz da Silva LD, Magalhães MTM, Pereira BKW, Pacheco RMT: Association between treatment compliance and different types of cardiovascular complications in arterial hypertension patients. Texto contexto enferm. 2016, $25: e 560015$. http://www.scielo.br/scielo.php?script=sci_arttext\&pid=S010407072016000300302 \&lng=en.

19. Mugwano I, Kaddumukasa M, Mugenyi L, et al.: Poor drug adherence and lack of awareness of hypertension among hypertensive stroke patients in Kampala, Uganda: a cross sectional study. BMC Res Notes. 2016, 9:3. 10.1186/s13104-015-1830-4 